An experimental and numerical investigation of the dynamics of microconfined droplets in systems with one viscoelastic phase

\author{
R. Cardinaels and P. Moldenaers \\ Lab for Applied Rheology and Polymer Processing \\ Department of Chemical Engineering \\ KU Leuven \\ Willem de Croylaan 46, Box 2423, B-3001 Leuven, Belgium \\ Paula.Moldenaers@cit.kuleuven.be \\ S. Afkhami, Y. Renardy \\ Department of Mathematics and ICAM \\ Virginia Polytechnic Institute and State University \\ 460 McBryde Hall, Blackburg, VA24061-0123, USA \\ Renardyy@aol.com
}

Final accepted draft

Cite as: R. Cardinaels, S. Afkhami, Y. Renardy, P. Moldenaers J. Non-Newt. Fluid Mech., 166 (1-2), pp. 52-62 (2011)

The original publication is available at:

http://www.sciencedirect.com/science/article/pii/S0377025710002697 


\section{Accepted Manuscript}

Title: An experimental and numerical investigation of the dynamics of microconfined droplets in systems with one viscoelastic phase

Authors: Ruth Cardinaels, Shahriar Afkhami, Yuriko Renardy, Paula Moldenaers

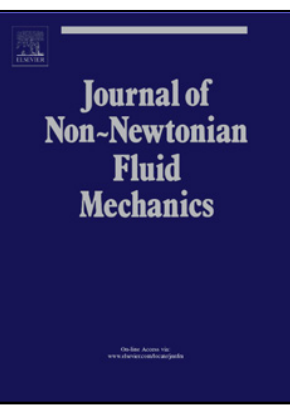

PII: S0377-0257(10)00269-7

DOI: doi:10.1016/j.jnnfm.2010.10.005

Reference: JNNFM 3151

To appear in: J. Non-Newtonian Fluid Mech.

Received date:

9-7-2010

Revised date:

8-10-2010

Accepted date: $11-10-2010$

Please cite this article as: R. Cardinaels, S. Afkhami, Y. Renardy, P. Moldenaers, An experimental and numerical investigation of the dynamics of microconfined droplets in systems with one viscoelastic phase, Journal of Non-Newtonian Fluid Mechanics (2010), doi:10.1016/j.jnnfm.2010.10.005

This is a PDF file of an unedited manuscript that has been accepted for publication. As a service to our customers we are providing this early version of the manuscript. The manuscript will undergo copyediting, typesetting, and review of the resulting proof before it is published in its final form. Please note that during the production process errors may be discovered which could affect the content, and all legal disclaimers that apply to the journal pertain. 


\title{
An experimental and numerical investigation of the dynamics of microconfined droplets in systems with one viscoelastic phase
}

\author{
Ruth Cardinaels $^{\mathrm{a}}$, Shahriar Afkhami ${ }^{\mathrm{b}}$, Yuriko Renardy ${ }^{\mathrm{c}, *}$, Paula Moldenaers $^{\mathrm{a}}$ \\ ${ }^{a}$ Katholieke Universiteit Leuven, Department of Chemical Engineering and Leuven Materials Research Center, W. de \\ Croylaan 46, B-3001 Heverlee, Belgium \\ ${ }^{b}$ Department of Mathematical Sciences, New Jersey Institute of Technology, University Heights, Newark, New Jersey \\ 07102-1982, USA \\ ${ }^{c}$ Department of Mathematics and ICAM, 460 McBryde Hall, Virginia Polytechnic Institute and State University, \\ Blacksburg VA 24061-0123, USA
}

\begin{abstract}
The dynamics of single droplets in a bounded shear flow is experimentally and numerically investigated for blends that contain one viscoelastic component. Results are presented for systems with a viscosity ratio of 1.5 and a Deborah number for the viscoelastic phase of 1 . The numerical algorithm is a volume-of-fluid method for tracking the placement of the two liquids. First, we demonstrate the validation of the code with an existing boundary integral method and with experimental data for confined systems containing Newtonian components. This is followed by numerical simulations and experimental data for the combined effect of geometrical confinement and component viscoelasticity on the droplet dynamics after startup of shear flow at a moderate capillary number. The viscoelastic liquids are Boger fluids, which are modeled with the Oldroyd-B constitutive model and the Giesekus model. Confinement substantially increases the viscoelastic stresses and the elongation rates in and around the droplet. We show that the latter can be dramatic for the use of the Oldroyd-B model in confined systems with viscoelastic components. A sensitivity analysis for the choice of the model parameters in the Giesekus constitutive equation is presented.

Keywords: droplet deformation, Oldroyd-B, numerical simulation, blend morphology, viscoelasticity, confinement
\end{abstract}

${ }^{*}$ Corresponding author

Email address: renardyy@gmail . com (Yuriko Renardy )

Preprint submitted to Journal of Non-Newtonian Fluid Mechanics

October 8, 2010 


\section{Introduction}

Technology improvements have triggered a continuous trend towards ever smaller scales. The ability to create structures and patterns on micron and smaller length scales has led to the use of microfluidic devices in a variety of applications $[1,2,3,4]$. When transporting complex twophasic fluids in microdevices, deviations from bulk behavior can be expected if the dimensions of the channel become comparable to the size of the dispersed phase $[1,3,5]$. Up to now, research on confined multiphase fluid dynamics has mainly been limited to systems that contain only Newtonian components [3,5]. Microfluidic applications are however diverse, ranging from life sciences industries for pharmaceuticals and biomedicine, to printing, microreaction engineering, etc. Therefore, the fluid rheology is an important factor in the design and optimization of microfluidic devices. Recently, there is a growing interest in the effect of viscoelasticity of one of the components on the dynamics of multiphase flows in microfluidic systems $[6,7,8,9]$.

In order to gain more fundamental insight in the physical phenomena that drive microstructure formation in confined multiphase flow, a number of studies have focused on droplet dynamics in blends with only Newtonian components that are placed in a confined shear flow. A recent review is given by Van Puyvelde et al. [5]. It is found experimentally, theoretically and numerically, that the presence of two parallel walls increases the steady state deformation and the orientation towards the flow direction for droplets in shear flow [5]. In addition, after startup of shear flow, confinement causes the steady state to be reached after longer times, especially at high viscosity ratios [10]. At very high confinement ratios and for capillary numbers close to the critical value, steady state is only reached after long-time oscillations of the droplet deformation and droplet orientation, that are not present in unconfined Newtonian blends [10, 11]. In addition, highly confined droplets become sigmoidal. For systems with Newtonian components, the full transient behavior of droplets in the whole range of confinement ratios and capillary numbers is well described by numerical simulations $[12,13,14,15]$.

In bulk shear flow, the effects of component viscoelasticity on the droplet dynamics have extensively been studied. Experimentally it was shown that the droplet deformation in a viscoelastic Boger fluid matrix is less as compared to that of droplets in a Newtonian matrix $[16,17,18,19,20]$. If the Boger fluid is used as the droplet fluid on the other hand, only minor effects of viscoelasticity have been observed [17, 20,21]. For high values of the flow intensity and for highly elastic materials, matrix viscoelasticity can induce considerable overshoots in the 
droplet deformation, accompanied by undershoots in the orientation angle [17, 18, 22]. Droplet viscoelasticity is less studied but it seems to have no major influence on the deformation dynamics [17, 20, 23]. Recently, researchers have started to experimentally investigate the combined effects of component viscoelasticity and geometrical confinement [16, 24, 25]. In addition, threedimensional numerical simulations of the droplet behavior in blends with viscoelastic fluids are emerging [17, 23, 26, 27, 28, 29, 30,31]. However, to our knowledge, no numerical studies of the droplet dynamics in confined shear flow have been performed yet for systems with viscoelastic components.

In this work, the effect of confinement on the droplet dynamics after startup of shear flow is studied for systems with one viscoelastic component. Experimental data obtained from droplet visualisation experiments in a counter rotating shear flow device are compared to the results of a volume-of-fluid simulation method. Pressure fields, stream lines as well as contours of the viscoelastic stresses, obtained from the numerical simulations, are used to gain physical insight in the droplet behavior.

\section{Materials and methods}

\subsection{Rheological characterization}

The deformation of droplets is microscopically studied for systems with either a viscoelastic droplet or a viscoelastic matrix. A Boger fluid (BF2, a complete rheological characterization of the material can be found in previous work $[17,18]$ ) consisting of $0.2 \mathrm{w} \%$ high molecular weight polyisobutylene (Oppanol B200) in a highly viscous polyisobutylene solvent (PIB, Infineum S1054) is used as the viscoelastic fluid throughout the study. The Newtonian phase consists of linear polydimethylsiloxanes (PDMS, Rhodorsil and Silbione) with different molecular weights, that were chosen to obtain a ratio $\lambda$ of droplet to matrix viscosity of 1.5 . When used as the matrix phase, PDMS was first saturated with low molecular weight PIB (Indopol H50) in order to avoid depletion of the low molecular weight fraction PIB in the droplet due to diffusion into the PDMS matrix fluid [32]. The system consisting of a Newtonian PDMS droplet in a Newtonian PIB matrix was used as the reference system. The interfacial tension $\Gamma$ of the different blends was determined by fitting the slow flow droplet deformation data to the second order theory of Greco [33]. The experimental temperatures and the corresponding characteristics of the experimental 
TABLE 1: Blend and component characteristics at viscosity ratio $\lambda=1.5$.

\begin{tabular}{ccccccccc}
\hline Blend & $\mathrm{d} / \mathrm{m}$ & Droplet & Matrix & $\mathrm{T}$ & $\eta_{m}$ & $\Psi_{1, d}$ & $\Psi_{1, m}$ & $\Gamma$ \\
& & & & ${ }^{\circ} \mathrm{C}$ & Pa.s & ${\text { Pa. } s^{2}}^{\text {Pa. } s^{2}}$ & $\mathrm{mN} / \mathrm{m}$ \\
\hline & & & & & & & & \\
1 & $\mathrm{~N} / \mathrm{N}$ & PDMS100-200 & PIB1300 & 25.5 & 83.5 & 0 & 0 & 2.7 \\
2 & $\mathrm{~N} / \mathrm{VE}$ & PDMS30-100 & BF2 & 26.4 & 36.5 & 0 & 197 & 2.0 \\
3 & $\mathrm{VE} / \mathrm{N}$ & $\mathrm{BF} 2$ & PDMS30 & 26.0 & 25.2 & 212 & 0 & 2.2 \\
\hline
\end{tabular}

system are given in Table 1. The properties of the matrix and droplet fluid are denoted with subscripts $\mathrm{m}$ and $\mathrm{d}$, respectively.

\subsection{Experimental setup}

The droplet dynamics in shear flow are experimentally studied by means of a counter rotating plate-plate device (based on a Paar Physica MCR300), equipped with a microscope (Wild M5A) and a digital camera (Basler 1394). The latter are mounted on vertically translating stages, enabling observations of the droplet shape in both the velocity/velocity gradient plane and the velocity-vorticity plane. The experimental setup and image analysis protocol are detailed elsewhere $[16,18]$.

\section{Numerical simulations}

\subsection{Governing equations}

The motion and deformation of a single droplet in a matrix fluid can be described by the momentum conservation equation and the incompressibility criterion for both fluids:

$$
\begin{aligned}
& \nabla \cdot \mathbf{u}=0 \\
& \rho\left(\frac{\partial \mathbf{u}}{\partial t}+(\mathbf{u} \cdot \nabla) \mathbf{u}\right)=\underset{4}{\nabla} \mathbf{T}-\underset{4}{\nabla}+\nabla \cdot\left(\eta_{s}\left(\nabla \mathbf{u}+(\nabla \mathbf{u})^{T}\right)\right)+\mathbf{F}
\end{aligned}
$$


where $\mathbf{u}$ denotes the velocity field, $\rho$ and $\eta_{s}$ the density and solvent viscosity of the fluid, $\mathrm{p}$ the pressure, $\mathbf{T}$ the extra stress tensor and $\mathbf{F}$ the interfacial tension force that is formulated as a body force:

$$
\mathbf{F}=\Gamma \tilde{\kappa} \mathbf{n} \delta_{s}
$$

with $\mathbf{n}$ the unit normal to the interface, $\delta_{s}$ the delta-function at the interface and $\tilde{\kappa}$ the curvature $-\nabla \cdot \mathbf{n}$ of the interface. Each liquid is represented by a color function,

$$
C(x, y, z, t)= \begin{cases}0 & \text { in the matrix } \\ 1 & \text { in the droplet, }\end{cases}
$$

which is advected by the flow. The position of the interface is reconstructed from the locations where $C(x, y, z, t)$ jumps from 0 to 1 , where $\mathbf{n}=\nabla \mathbf{C} /|\nabla \mathbf{C}|$ and $\delta_{S}=|\nabla C|$. For the extra stress tensor $\mathbf{T}$, the Giesekus constitutive equation is used:

$$
\tau_{p}\left(\frac{\partial \mathbf{T}}{\partial t}+(\mathbf{u} \cdot \nabla) \mathbf{T}-(\nabla \mathbf{u}) \mathbf{T}-\mathbf{T}(\nabla \mathbf{u})^{T}\right)+\mathbf{T}+\frac{\tau_{p} \alpha}{\eta_{p}} \mathbf{T}^{2}=\eta_{p}\left(\nabla \mathbf{u}+(\nabla \mathbf{u})^{T}\right)
$$

where $\tau_{p}$ is the relaxation time of the fluid, $\eta_{p}$ the polymer part of the zero-shear rate viscosity and $\alpha$ the mobility parameter of the Giesekus model. A value $\alpha=0$ results in the Oldroyd-B constitutive equation.

Buoyancy and inertia effects can be neglected in the experiments. Therefore, the numerical simulations are performed with density-matched liquids and the Reynolds number Re (= $\rho \dot{\gamma} R^{2} / \eta_{m}$, with $\dot{\gamma}$ the shear rate and $\mathrm{R}$ the droplet radius) is chosen small $(\mathrm{Re}=0.1)$ so that inertia is negligible. The remaining dimensionless parameters that govern the behavior of a Newtonian droplet in a Newtonian matrix are the viscosity ratio $\lambda=\eta_{d} / \eta_{m}$ and the capillary number $\mathrm{Ca}$ $=\left(R \dot{\gamma} \eta_{m}\right) / \Gamma$. If the viscoelastic fluid is described by the Oldroyd-B model, two additional dimensionless groups should be included. The retardation parameter $\beta=\eta_{s} / \eta$ gives the solvent part of the viscosity of the viscoelastic fluid. In addition, a Weissenberg number $\mathrm{Wi}=\dot{\gamma} \tau_{p}$ is defined. For the Giesekus model, the dimensionless Giesekus parameter $\alpha$ is needed to fully specify the material rheology. The latter determines the predictions of the Giesekus model for both the second normal stress difference and the high-shear plateau value of the Trouton ratio Tr:

$$
\alpha=\frac{-2 \Psi_{2}}{\Psi_{1}}=\frac{2(1-\beta)}{\operatorname{Tr}}
$$

with $\Psi_{1}$ and $\Psi_{2}$ the first and second normal stress coefficients. After setting all previous parameters, the Deborah number, $\mathrm{De}=\left(\Psi_{1} \Gamma\right) /\left(2 \mathrm{R} \eta^{2}\right)$, which describes the ratio of the fluid relaxation 
time $\tau_{p}$, based on a second-order fluids model $\left(\tau_{p}=\Psi_{1} / 2 \eta\right)$, to the emulsion time $\tau_{E}=\left(\eta_{m} \mathrm{R} / \Gamma\right)$ of the droplet, is fixed since $D e_{d}=\left(1-\beta_{d}\right) \mathrm{Wi} /(\lambda \mathrm{Ca})$ and $D e_{m}=\left(1-\beta_{m}\right) \mathrm{Wi} / \mathrm{Ca}$. Viscoelastic effects on the droplet behavior become noticeable if the De number becomes order of magnitude 1 $[11,17,23,33]$. In the present work, all experiments and simulations are performed at $\mathrm{De}=1$.

\subsection{Numerical method}

The direct numerical simulations are performed with a volume-of-fluid (VOF) formulation which is detailed in literature $[17,23,29,34,35,36]$. Briefly, the code uses a finite difference methodology on a regular Cartesian mesh. The placement of each fluid is determined from the discretized color function, which gives the volume fraction for the droplet liquid in each grid cell. The interface shape is reconstructed from the volume fraction function with the paraboloid reconstruction scheme (PROST) [29, 37], and it is advected in a Langrangian manner by the computed velocity field. The computational domain is a rectangular box with $0 \leq \mathrm{x} \leq L_{x}, 0 \leq$ $\mathrm{y} \leq L_{y}$ and $0 \leq \mathrm{z} \leq L_{z}=1$, where the velocity of the background shear is in the $\mathrm{x}$-direction and the velocity gradient is in the $\mathrm{z}$-direction. Spatial periodicity is imposed in the $\mathrm{x}$ and $\mathrm{y}$ directions. The value of the droplet radius $R_{0}$ is set to obtain the same confinement ratio $2 R_{0}=$ $2 \mathrm{R} / \mathrm{H}$ as in the experiments, $L_{x}$ and $L_{y}$ are chosen to minimize the effect of neighbouring droplets and the walls, typically $L_{x}$ and $L_{y}$ range from 1 to 4 , where higher values are used at higher confinement ratios. Convergence tests for spatial and temporal refinement were conducted for $D e_{m}=1, C a=0.2,2 R_{0} / H=0.44$. The results were independent of mesh size when $\Delta \mathrm{x}=\Delta \mathrm{y}=$ $\Delta \mathrm{z}=1 / 64$ and $\dot{\gamma} \cdot \Delta t=0.0001$. Therefore, further results are presented at this refinement. The initial condition consists of a spherical droplet in a matrix with zero initial viscoelastic stresses. The movement of the walls is started instantaneously and the velocity field adjusts immediately to a simple shear flow.

Droplet deformation in the velocity-velocity gradient plane (x-z plane) is quantified by means of the deformation parameter $\mathrm{D}=(\mathrm{L}-\mathrm{B}) /(\mathrm{L}+\mathrm{B})$, where $\mathrm{L}$ and $\mathrm{B}$ are the long and short droplet axes, respectively. In addition, the droplet orientation angle $\theta$ is defined as the angle between the longest axis of the droplet and the flow direction. For non-ellipsoidal droplets, the droplet deformation is characterized by means of $L_{p}$, which is the projection of the longest axis of the droplet on the velocity direction $\mathrm{x}$ and $W_{p}$, which is the droplet axis in the vorticity direction y. Generally, droplet axes are obtained by fitting an ellipse to the droplet contour in the $\mathrm{x}-\mathrm{z}$ and $x-y$ planes. Only for the highest confinement ratios, where the droplet shape starts to become 
sigmoidal, the droplet axes are taken to be twice the distance from the center to the farthest node on the droplet contour. In the following, we present $L_{p}$, and $W_{p}$ as dimensionless values, with respect to the initial droplet diameter.

\subsection{Benchmark computation and validation}

Simulations of droplet deformation in systems with one viscoelastic component are mostly available for unbounded domains, and the performance of VOF-PROST is documented in several works [17, 23, 26]. For example, VOF-PROST predictions agree with the results of Aggarwal and Sarkar [27, 38], who developed a numerical methodology based on front-tracking for the 3D study of droplet dynamics in blends with viscoelastic components. Their results on systems of equiviscous Oldroyd-B liquids in unbounded flow agree with those obtained with the VOFPROST code that is used in the present paper [26]. A comparison between numerical simulations with the VOF method and experimental data for droplet deformation and droplet orientation in bulk shear flow for systems with one viscoelastic component can be found in the work of Verhulst et al. [17, 23].

Validation of the numerical results for a confined droplet was performed for the case of a Newtonian liquid pair at $C a=0.35,2 R / H=0.88$ and $\lambda=1$. This set of parameters was chosen in order to compare with Figure 11 of Vananroye et al. [15], where numerical simulations using the boundary integral method (BIM) of Janssen et al. [12] are compared with experimental data for highly confined Newtonian droplets at viscosity ratio 1 . We also compare with the VOFbased height-function (VOF-HF) method [39, 40], which uses the oct-tree adaptive mesh. Fig. 1 shows the comparison of results with VOF-PROST simulations at the level of discretization that is used for the present paper, and with the VOF-HF method. Fig. 1(a) displays the dimensionless length $L_{p}(\circ)$ and width $W_{p}(\square)$ in the velocity-vorticity plane (z-y plane) versus dimensionless time $t / \tau_{E}$. Fig. 1(b) shows droplet shapes in the velocity - velocity gradient (x-z) and velocity - vorticity (z-y) planes and pressure fields obtained with the VOF-PROST method at $t / \tau_{E}=2$, 18.2, 60.6 and 135.5. The resolution is chosen at $\Delta x=\Delta y=\Delta z=H / 64, \Delta t \cdot \dot{\gamma}=0.001$, and the computational domain size is $L_{x}=4 H, L_{y}=2 H, L_{z}=H$. Fig. 1(a) shows that all three numerical results are close to each other. BIM and VOF-PROST overpredict the experimental values of $L_{p}$ with maximum deviations of $7 \%$ and $9 \%$, respectively, while the steady state $L_{p}$ is overpredicted by $3 \%$ and $5 \%$, respectively. A similar overprediction of the experimental data for highly confined droplets with numerical simulations can be found in literature for BIM [15] 
(a)
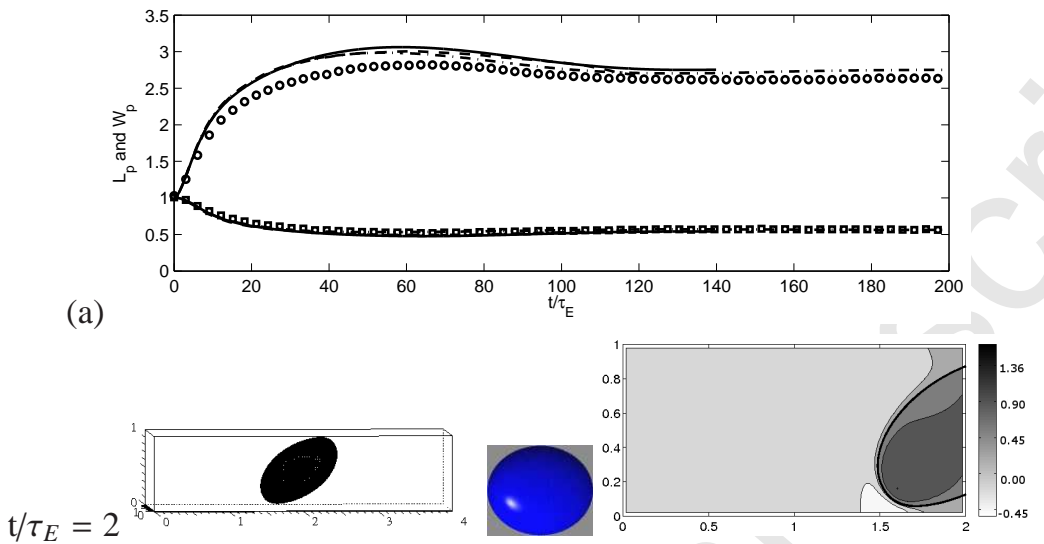

18.2
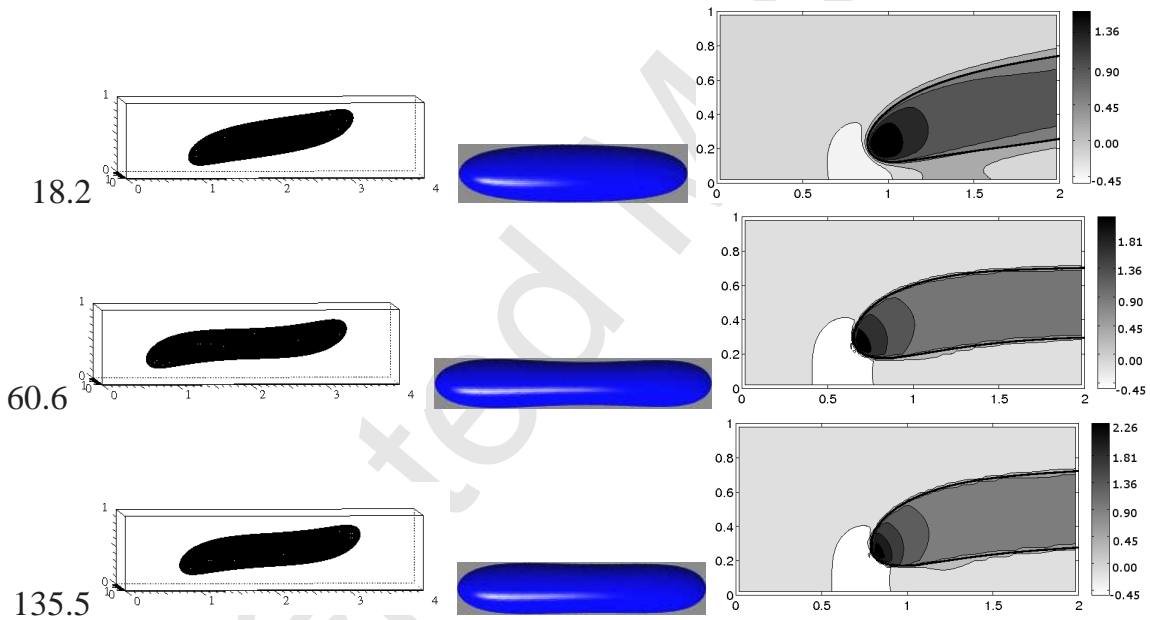

(b)

FIG. 1: (a) Length $L_{p}(\circ)$ and width $W_{p}(\square)$ in the velocity-vorticity plane versus $t / \tau_{E}$. Newtonian system, $C a=$ $0.35,2 R / H=0.88$, viscosity ratio 1 . Experimental data (symbols), BIM (-.-), VOF-PROST (-) and VOF-HF (- -), (b) Shapes (VOF-PROST) in the velocity - velocity gradient and velocity - vorticity planes and pressure fields relative to the Laplace pressure at $t / \tau_{E}=2,18.2,60.6$ and 135.5. 
and VOF [31]. The VOF-HF result lies between these methods. The experimentally obtained $W_{p}$ is consistently close to all three numerical simulations. The sigmoidal droplet shapes and the significant increase of the pressure at the droplet tip, that can be seen in Fig. 1(b), is in agreement with the results of the BIM method [41].

\section{Results and discussion}

\subsection{Startup dynamics of a viscoelastic droplet in a Newtonian matrix}

The evolution of the droplet deformation and orientation after startup of shear flow at $\mathrm{Ca}=$ 0.2 is shown in Fig. 2 for a viscoelastic droplet in a Newtonian matrix. Results are given for confinement ratios $2 \mathrm{R} / \mathrm{H}$ ranging from 0.11 to 0.74 . Time after startup of the flow is made dimensionless with the emulsion time $\tau_{E}$. The experimental results show that, from a dimensionless time of about 3 , confinement accelerates the droplet dynamics. In addition, it leads to more oriented and more deformed droplets than bulk shear flow at the same capillary number, as shown by Cardinaels et al. [16]. The results of VOF simulations, using the Oldroyd-B model to describe the droplet rheology, are also shown in Fig. 2. The parameters $\eta, \beta$ and $\tau_{P}$ in the Oldroyd-B model have been obtained from a best fit of the steady shear data of the Boger fluid, as in Table 3 in the work of Verhulst et. al [42]. The agreement between the experimental data and the results of VOF simulations is good, both for the deformation parameter and for the orientation angle. In addition, simulation results for blends with Newtonian components are added for the highest confinement ratio of 0.74 . The results of the VOF simulations for blends with a viscoelastic or a Newtonian droplet and the experimental results at $2 \mathrm{R} / \mathrm{H}=0.74$ almost coincide, indicating that, under these conditions, droplet viscoelasticity has a minor effect on the droplet deformation after startup of shear flow, even for confined droplets.

The pressure field in and around the droplet is shown in Fig. 3 for an unbounded and a confined droplet in steady shear flow at $\mathrm{Ca}=0.2$. The pressures are scaled with the Laplace pressure $2 \Gamma / \mathrm{R}$ for a spherical droplet. Increasing the confinement ratio results in an increase of the maximum pressure inside the droplet tip whereas in regions with low curvature, such as the droplet waist, the pressure remains low. This is in agreement with the results of Vananroye et al. [41] for systems with Newtonian components. From Fig. 3 it can be seen that the position of the pressure maximum shifts closer to the droplet tip when the droplet is confined. At this tip, the increased 


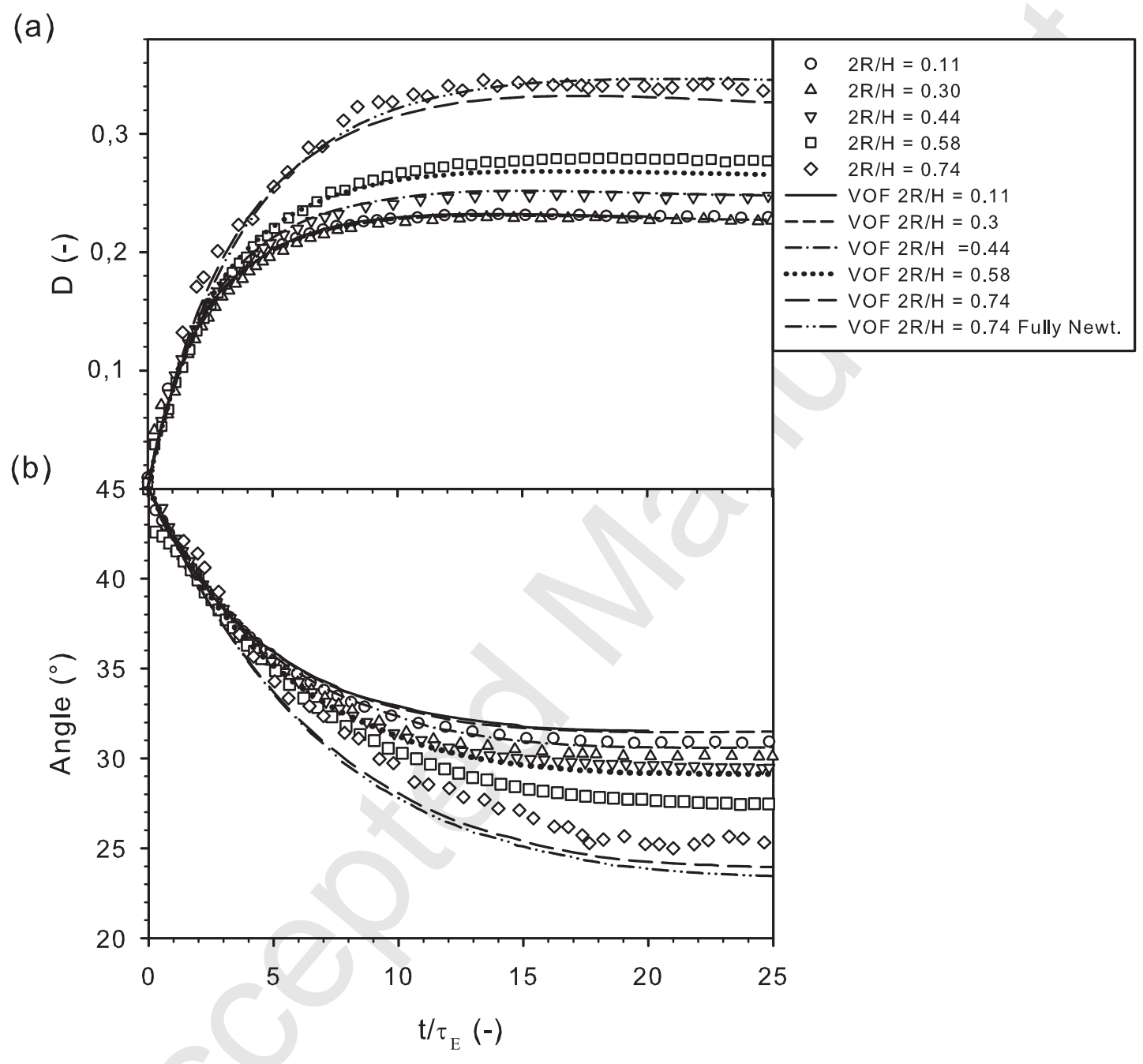

(a)

FIG. 2: Droplet dynamics after startup of shear flow at $\mathrm{Ca}=0.2$ for a viscoelastic droplet in a Newtonian matrix with $\lambda=$ 1.5. Comparison between experimental data (symbols), VOF simulations (lines) for systems with an Oldroyd-B droplet fluid $(\beta=0.68)$ and for systems with only Newtonian components. (a) Deformation parameter. (b) Orientation angle. 
pressure drop across the interface balances the high curvature that is present due to the more deformed droplet shape at high confinement ratios.

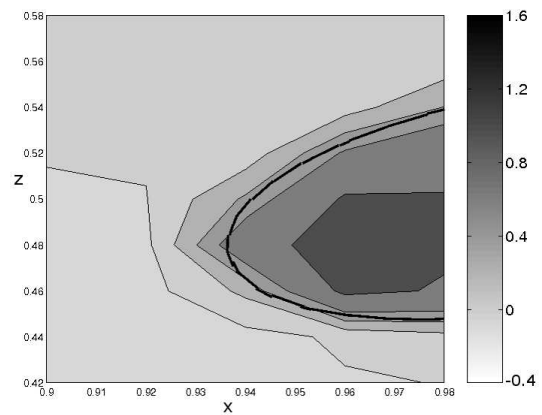

(a) $2 \mathrm{R} / \mathrm{H}=0.11$

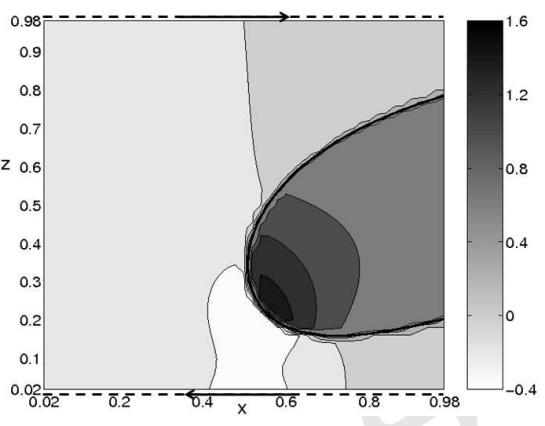

(b) $2 \mathrm{R} / \mathrm{H}=0.74$

FIG. 3: Pressure field in the $\mathrm{x}-\mathrm{z}$ cross-section, relative to the Laplace pressure of a spherical droplet. The motion is driven by moving walls at $\mathrm{z}=0$ and $\mathrm{z}=1$. Viscoelastic droplet in a Newtonian matrix at $\mathrm{Ca}=0.2$ and $\lambda=1.5$.

The stream lines in and around the droplet at $\mathrm{Ca}=0.2$ are shown in Fig. 4. In bulk conditions, the flow field is completely rotational, which is in agreement with earlier studies for systems with viscoelastic droplets $[17,27]$. However, also for the confined droplet, the droplet shape remains close to ellipsoidal and the flow field is almost a pure rotation. However, outside the droplet a large recirculation zone is formed at the front and rear of the droplet, together with higher shear rates below the droplet tip. This reversal of a substantial part of the fluid stream has also been reported for confined droplets in blends with Newtonian components [10, 12, 31]. For the present system, the extent of this recirculation zone increases from about $20 \%$ of the droplet height in bulk conditions to above $50 \%$ of the droplet height in confined conditions $(2 \mathrm{R} / \mathrm{H}=0.74)$. The changes in the flow field around the droplet, caused by confinement, might significantly alter droplet interactions and droplet coalescence in more concentrated or confined blends [43].

Fig. 5 gives the contour plots of the viscoelastic stress for different confinement ratios. In the present work, the trace of the extra stress tensor $\mathbf{T}$ is used to discuss the viscoelastic effects, since this quantity is directly proportional to the extension of the polymer molecules. The viscoelastic stress inside the droplet is mainly concentrated above the droplet tip at the back of the droplet and below the droplet tip at its front. It can be seen in Figs. 5(a) - 5(c) that with increasing confinement ratio, the maximum viscoelastic stress inside the droplet increases, showing that viscoelasticity of the droplet fluid becomes more important for confined droplets. However, 


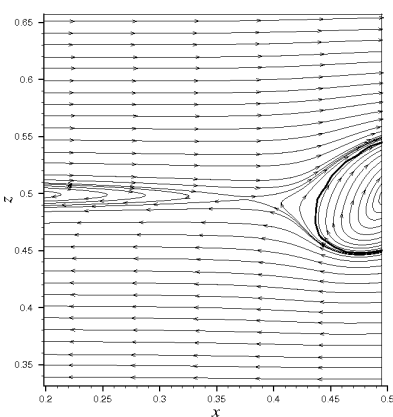

(a) $2 \mathrm{R} / \mathrm{H}=0.11$

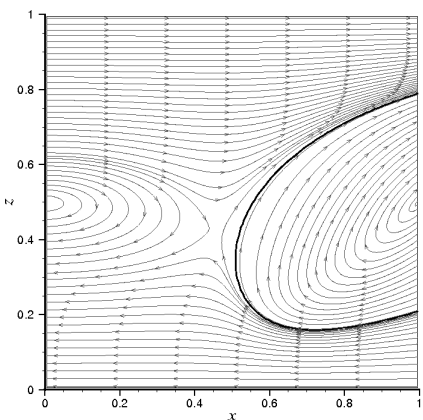

(b) $2 \mathrm{R} / \mathrm{H}=0.74$

FIG. 4: Stream lines in the $\mathrm{x}-\mathrm{z}$ cross-section. Viscoelastic droplet in a Newtonian matrix at $\mathrm{Ca}=0.2$ and $\lambda=1.5$.

the position of the stress maximum remains approximately the same. From the rather good agreement between the numerical results for the system with Newtonian components and the system with a viscoelastic droplet at $2 \mathrm{R} / \mathrm{H}=0.74$ (Fig. 2), it can be concluded that the increase of the viscoelastic stress is only very moderately reflected in the droplet deformation. This behavior is similar to that observed for increasing values of the De-number in bulk conditions [17]. In summary, the localized high viscoelastic stress in confined conditions might slightly influence the details of the shape of the droplet tip, but it has an insignificant effect on the overall droplet deformation.

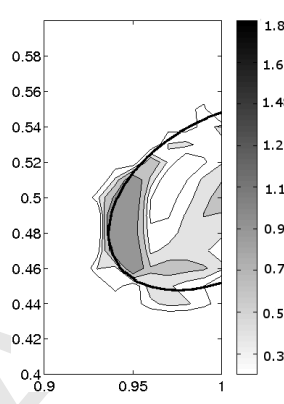

(a) $2 \mathrm{R} / \mathrm{H}=0.11$

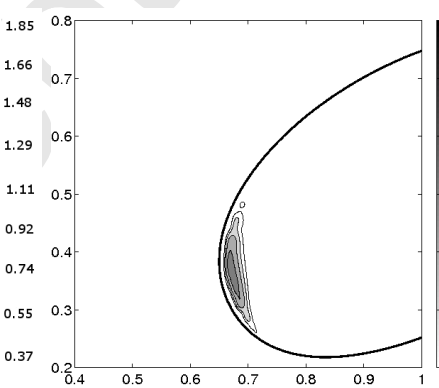

(b) $2 \mathrm{R} / \mathrm{H}=0.58$

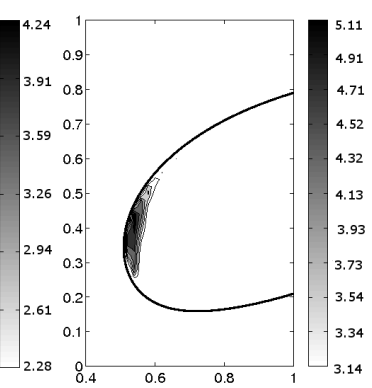

(c) $2 \mathrm{R} / \mathrm{H}=0.74$

FIG. 5: Contour plots of the trace (T) in the $\mathrm{x}-\mathrm{z}$ cross-section, stresses are in Pa. Viscoelastic droplet in a Newtonian matrix at $\mathrm{Ca}=0.2$ and $\lambda=1.5$. 


\subsection{Startup dynamics of a Newtonian droplet in a viscoelastic matrix}

\subsubsection{Droplet deformation and droplet orientation}

The experimental results for the deformation parameter and the orientation angle of a Newtonian droplet in a viscoelastic matrix are presented in Fig. 6 for a series of confinement ratios. For confinement ratios $2 \mathrm{R} / \mathrm{H}$ up to 0.75 , a monotonous evolution towards the steady values is obtained. Similar to the results for a viscoelastic droplet, shown in Fig. 2, confinement accelerates the deformation kinetics and increases the steady state droplet deformation and droplet orientation. In addition, the dimensionless time at which confinement starts to affect the droplet behavior is approximately the same, independent of which phase is viscoelastic (Figs. 2 and 6). However, from a comparison between the steady state values of D in Figs. 2 and 6 it is clear that, for all confinement ratios, a Newtonian droplet in a viscoelastic matrix is less deformed than a viscoelastic droplet in a Newtonian matrix. Therefore, the presence of viscoelastic stresses is clearly manifested in the results for droplets in a viscoelastic matrix that are presented in Fig. 6.

The results of simulations with the VOF method have been added to Figs. 6(a) and 6(b) for different confinement ratios. Analogous to the simulations for blends with a viscoelastic droplet, the Oldroyd-B model was used to describe the rheology of the Boger fluid matrix. Under bulk conditions, this method has proven to be successful as long as the transient droplet deformation follows a monotonous startup transient [17]. A comparison between the simulation results for the droplet deformation and the experimental results indicates that up to a confinement ratio of 0.44 the agreement is satisfactory. However, if the confinement ratio is increased to 0.60 or 0.75 , the numerical simulations capture the initial trend of the droplet dynamics, but overpredict the steady droplet deformation. Fig. 6(a) shows that the deviation between the experimental and the simulated deformation gradually increases with confinement ratio and the simulations with an Oldroyd-B matrix result in a higher value for the droplet deformation than the experimental

one. For the orientation angle, there is already a discrepancy at low confinement ratios. The simulation results for systems with Newtonian components are included in Figs. 6(a) and 6(b) for a confinement ratio $2 \mathrm{R} / \mathrm{H}$ of 0.75 . The simulated curve for the system with a viscoelastic matrix shows a slightly slower transient deformation than that for the system with Newtonian components. This is in agreement with the results obtained by previous authors for the evolution of the droplet deformation in a viscoelastic matrix after startup of bulk shear flow [38, 44, 45]. 


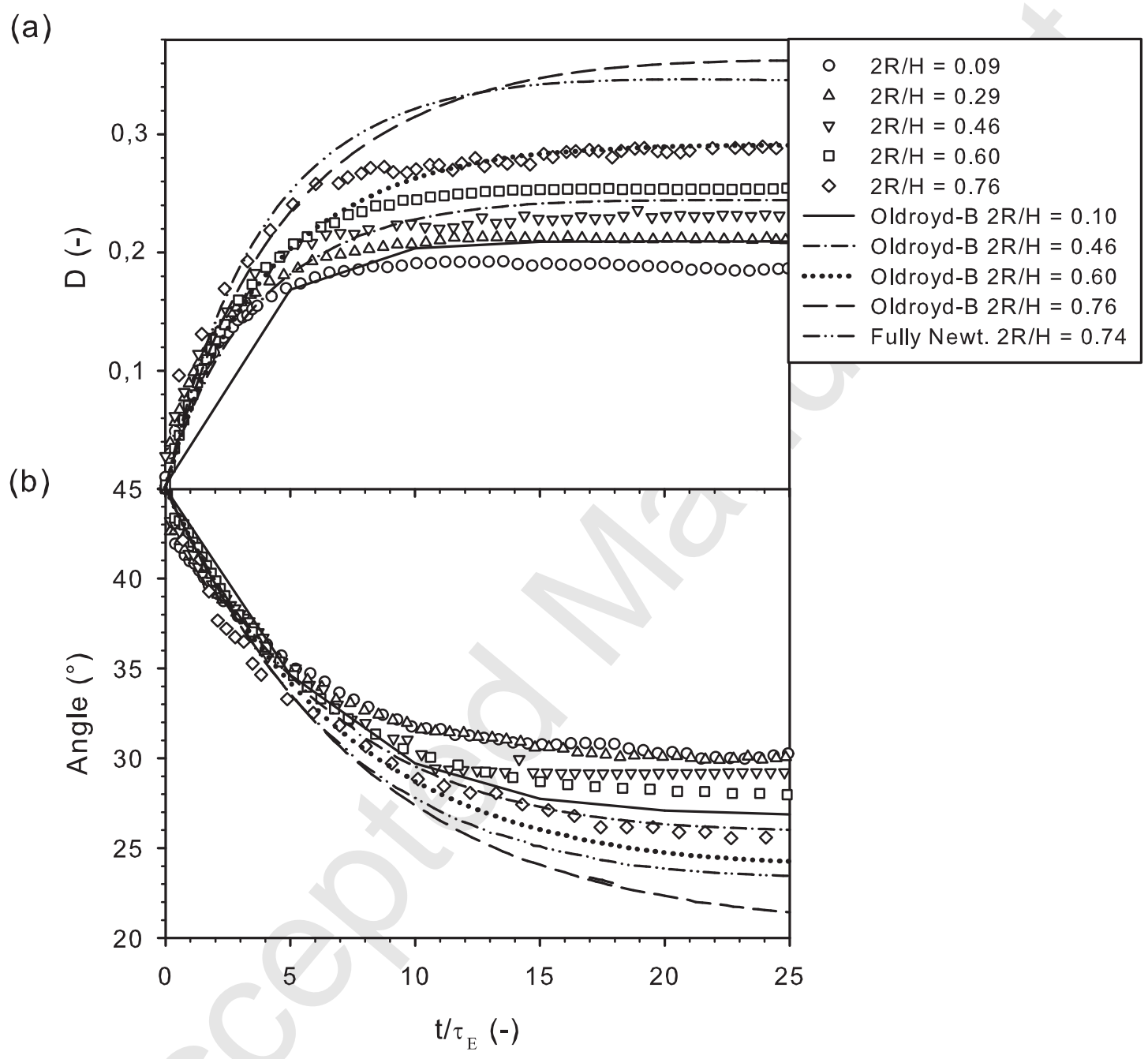

(a)

FIG. 6: Droplet dynamics after startup of shear flow at $\mathrm{Ca}=0.2$ for a Newtonian droplet in a viscoelastic matrix with $\lambda$ $=1.5$. Experimental data (symbols) and VOF simulations (lines) for systems with an Oldroyd-B matrix fluid $(\beta=0.68)$ and for systems with only Newtonian components. (a) Deformation parameter, (b) Orientation angle. 


\subsubsection{Sensitivity analysis for the parameters in the constitutive equation for the matrix fluid}

From a comparison between Figs. 2 and 6 it can be seen that the bulk droplet deformation in an Oldroyd-B matrix with $\mathrm{De}=1$ is smaller than that in a Newtonian matrix $(\mathrm{D}=0.21$ as compared to 0.23 ). However, at the highest confinement ratio shown in Fig. 6, the simulations for systems with an Oldroyd-B matrix give a value for the droplet deformation that is higher than that of droplets in a Newtonian matrix. An increase of the droplet deformation due to the viscoelasticity of the Oldroyd-B matrix fluid has also been observed with diffuse-interface and front-tracking finite difference simulation methods for unbounded droplets in high Weissenberg number flows $[38,46]$. However, experiments with a viscoelastic Boger fluid matrix generally show a reduction of the droplet deformation $[17,18,19,20]$. Therefore, under more critical conditions such as high values of $\mathrm{Wi}$ or high confinement ratios, the numerical results for systems with an Oldroyd-B matrix deviate from the experimental results for blends with a Boger fluid matrix. The deviation is more pronounced as $\mathrm{Ca}$ and/or De grows larger.

A possible explanation for this mismatch can be found by studying the elongation rates around the droplet. The Oldroyd-B model predicts an infinite value of the elongational viscosity at a dimensionless elongation rate $\left(\dot{\varepsilon} \cdot \tau_{P}\right)$ of 0.5 [47]. Therefore, numerical problems can be expected if the dimensionless elongation rates in and around the droplet exceed this critical value. In order to quantify this, we define the elongation rate to be the largest positive eigenvalue of the $2 \times 2$ matrix

$$
\left(\begin{array}{ll}
\frac{\partial u}{\partial x} & \frac{\partial u}{\partial z} \\
\frac{\partial w}{\partial x} & \frac{\partial w}{\partial z}
\end{array}\right)
$$

where we consider the $\mathrm{x}-\mathrm{z}$ cross-section at the droplet center and $\mathrm{u}$ and $\mathrm{w}$ are the velocities in the $\mathrm{x}$ and $\mathrm{z}$ directions respectively. The evolution of the maximum dimensionless elongation rate around the droplet at three different confinement ratios is shown as a function of time in Fig. 7. It is clear from this figure that the dimensionless elongation rate remains below the critical value at a confinement ratio of 0.09 , but it exceeds this value for higher confinement ratios. At a confinement ratio of 0.76 , it crosses the critical value of 0.5 at a dimensionless time as low as 3 . The simulated values for the deformation parameter on the other hand agree with the experimental results up to a dimensionless time of about 7 (Fig. 6(a)). This can be attributed to the fact that the elongational viscosity needs sufficient time to build up in order to influence the droplet behavior, as was shown in Figure A.2 of the work of Verhulst et al. [17]. The contours of 
$\operatorname{tr}(\mathbf{T})$ at $\mathrm{t} / \tau_{E}=10$ show that there is a region just outside the interface where viscoelastic stresses build up. The streamlines are shown at the same time, to illustrate the existence of a hyperbolic streamline roughly at the edge of the region where stresses are growing. With time, the gradient of $\operatorname{tr}(\mathbf{T})$ grows in the direction of the dividing streamline. This is reminiscent of the result of Ref. [48] which establishes the formation of singular structures in the polymer stress field at hyperbolic stagnation points in the flow of an Oldroyd-B fluid. The stresses grow exponentially in time along a dividing streamline. In our case, although the maximum elongation rate is a small distance downstream of the stagnation point, the manner in which the viscoelastic stresses blow up depends on the elongation rate at the stagnation point itself; this is difficult to resolve numerically. Therefore, we study the maximum elongation rate, but we need to keep in mind that the quantity of interest is less than that. For example, at $2 \mathrm{R} / \mathrm{H}=0.46$, the maximum elongation rate in Fig. 7 is above 0.5, while the agreement in Fig. 6 is still reasonable, and this can be attributed to the fact that the elongation rate at the stagnation point is somewhat lower than the maximum value. In our simulations, we see numerically that the elastic energy, represented by $\operatorname{tr}(\mathbf{T})$ grows to unphysically large values when the product of elongation rate and relaxation time is above roughly 0.5 . Beyond this value, the prediction of the Oldroyd-B model for the elongational viscosity breaks down. At this time, a numerical resolution to this issue is not clear. One method is to use a model that is nonlinear in the stress tensor and therefore we choose the Giesekus model which has an additional parameter $\alpha$ and thus enables the modeling of a finite elongational viscosity at high elongation rates.

Numerical simulations have been performed in which the rheology of the matrix fluid was represented by the Giesekus constitutive equation. From Eq. 6 it can be seen that the predictions of the Giesekus model for the elongational viscosity depend mainly on the value of the dimensionless Giesekus parameter $\alpha$. Therefore, the dimensionless Giesekus parameter was varied in order to assess the effect of the elongational viscosity on the droplet behavior. Initially, $\alpha=0.002$ was chosen, which results in a plateau Trouton ratio of 320 . This value is much lower than the experimental Trouton ratio that is approximately $3.10^{4}$ [17]. The result of a simulation with this value of $\alpha$ is shown in Table 2(a) at a confinement ratio of 0.76 . The deformation parameter (and also the orientation angle) for a droplet in an Oldroyd-B matrix and a droplet in a viscoelastic matrix that obeys the Giesekus model with $\alpha=0.002$ are exactly the same. An increase of the mobility parameter $\alpha$ only results in a reduction of the droplet deformation for considerably 

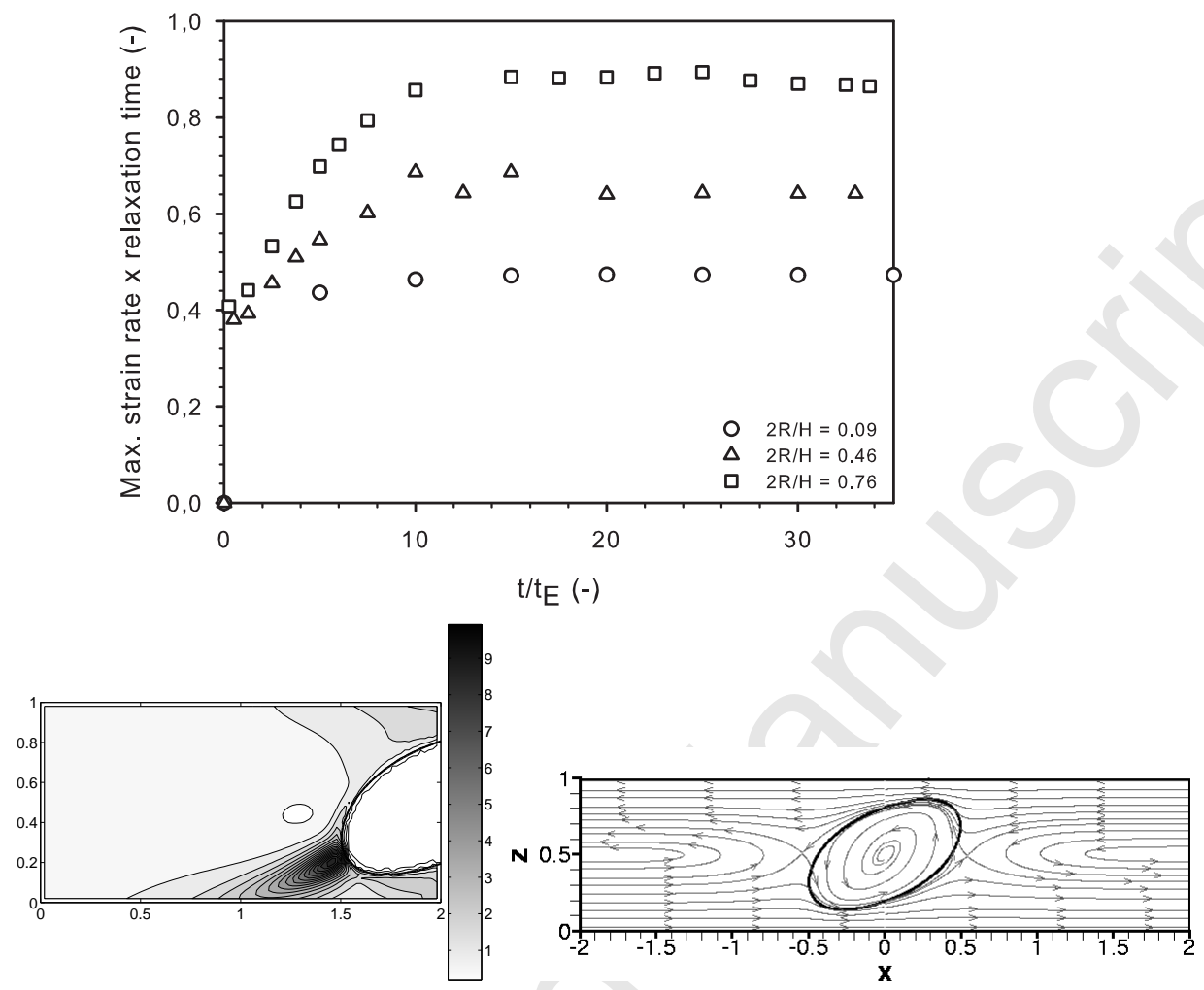

FIG. 7: Newtonian droplet in a viscoelastic matrix with $\lambda=1.5$ after startup of shear flow at Ca $=0.2$. Evolution of the maximum of the dimensionless elongation rate $\left(\dot{\varepsilon} \cdot \tau_{P}\right)$ as a function of time in VOF simulations for an Oldroyd-B matrix with $\beta=0.68$. For $2 \mathrm{R} / \mathrm{H}=0.76$ and $\mathrm{t} / \tau_{E}=10$, contours of the $\operatorname{tr}(\mathbf{T})$ and streamlines are shown. The values of $\operatorname{tr}(\mathbf{T})$ reflect the propagation of elastic energy, with the gradient in the direction of the dividing streamlines.

higher values of $\alpha$, where the elongational viscosity has dropped to a value that is of the same order of magnitude as the shear viscosity ( $\alpha \geq 0.1$, Eq. 6). However, even when the limiting value for obtaining physically sound results $(\alpha=0.5)$ is almost reached [49], the simulation results slightly overpredict the experimental data (Table 2(a)). In addition, as shown in Eq. 6, the value of $\alpha$ also determines the ratio of the second to the first normal stress difference. For Boger fluids, this ratio is known to be very low [50]. However, the second normal stress difference in polymer melts has been shown to influence the droplet deformation [51]. Therefore, using a too high value for $\alpha$ in the simulations might result in additional contributions of the second normal stress difference, that are not present in the experimental system. Therefore, in the remainder of 
this work, the value of $\alpha$ is limited to a maximum of 0.2 , which agrees with a ratio $-\Psi_{2} / \Psi_{1}$ of 0.1 , in agreement with literature values for polymer solutions and Boger fluids [50, 52, 53, 54, 55].

In addition to the dimensionless Giesekus parameter $\alpha$, the fluid rheology represented by the Giesekus model is determined by the solvent contribution to the viscosity $\beta$ and by the polymer relaxation time $\tau_{P}$. The rheology of the Boger fluid matrix material is far more complex than that of an Oldroyd-B or Giesekus fluid with a single relaxation time. The steady and dynamic shear rheology and the elongational viscosity of the Boger fluid BF2 can be well described by means of a 5-mode Giesekus model [42]. A substantial amount of the polymer contribution to the viscosity has a relaxation time $\tau_{P}$ that is far below the emulsion time $\tau_{E}$, which is on the order of 3 seconds in the present work. Thus, these modes do not contribute to the viscoelastic response at the time scales that are of relevance here. Therefore, a more representative single-mode model would consist of an Oldroyd-B or Giesekus fluid with a lower polymer contribution and a higher average relaxation time. In order to assess the influence of the solvent contribution, simulations were performed for systems with an Oldroyd-B or Giesekus matrix with a solvent contribution $\beta$ of 0.84 as compared to 0.68 in the previous simulations. In addition, the relaxation time and consequently also the Weissenberg number were increased whereas the Deborah number was kept constant at 1 . In bulk shear flows with a high Wi number, an increase of $\beta$ can relief the deforming viscoelastic stresses somewhat and reduce the droplet deformation [17, 38]. However, the results in Table 2(a) for $2 \mathrm{R} / \mathrm{H}=0.76$ indicate that for an Oldroyd-B matrix with $\beta=0.84$ an even higher droplet deformation is obtained as compared to that for droplets in an Oldroyd-B matrix with $\beta=0.68$. Therefore, under the present conditions, the increase of the Weissenberg number and the corresponding increase of the extension of the polymer molecules overtake the reduction in polymer content in the Boger fluid.

From Table 2(b) it can be seen that the results for simulations with the Oldroyd-B model or the Giesekus model with $\alpha=0.2$ coincide, up to the confinement ratio where the elongation rate exceeds the critical value. This supports the above proposed explanation for the overestimation of the droplet deformation. However, for the highest confinement ratio, an exact quantitative agreement with the experimental data is still not obtained. Therefore, it can be concluded that in confined systems, the complex rheology and multiple relaxation times of the Boger fluid can not be lumped easily into a simple single-mode model. This conclusion is in line with the results for the deformation of unconfined droplets in a viscoelastic matrix in bulk shear flow [17, 23]. 
However, in bulk conditions deviations between experimental and simulation results were only observed when the droplet deformation overshoots before reaching its final steady state value. From the results in Table 2(b) it can be concluded that the droplet deformation becomes more sensitive to the exact viscoelastic nature of the matrix fluid when geometrical confinement comes into play. This is most probably caused by the more complex flow field in confinement, as shown in Fig. 4, that involves a larger variation in type and intensity of the flow around the droplet. 
TABLE 2: Steady state deformation parameter $\mathrm{D}$ at $\mathrm{Ca}=0.2$ for a Newtonian droplet in a viscoelastic matrix with $\lambda=$ 1.5. A sensitivity analysis of the VOF simulations to changes in the model parameters.

(a) $2 \mathrm{R} / \mathrm{H}=0.76$

Experimental

0.29

Oldroyd-B $\beta=0.68 \alpha=0$

0.36

Giesekus $\beta=0.68 \alpha=0.002 \quad 0.36$

Giesekus $\beta=0.68 \alpha=0.4 \quad 0.31$

Giesekus $\beta=0.84 \alpha=0 \quad 0.37$

Giesekus $\beta=0.84 \alpha=0.2 \quad 0.33$

(b) $2 \mathrm{R} / \mathrm{H} \quad$ Exp. Oldroyd-B Giesekus

\begin{tabular}{cccc} 
& & $\beta=0.68$ & $\beta=0.84$ \\
& & $\alpha=0$ & $\alpha=0.2$ \\
\hline 0.09 & 0.19 & 0.21 & 0.19 \\
0.46 & 0.23 & 0.24 & 0.23 \\
0.60 & 0.25 & 0.29 & 0.27 \\
0.76 & 0.29 & 0.36 & 0.33 \\
\hline
\end{tabular}




\subsubsection{Viscoelastic stresses and elongation rates in and around the droplet}

In order to qualitatively study the effect of confinement on the viscoelastic stresses, the flow field and the pressure in and around a deformed droplet in a viscoelastic matrix, the contour plots of these variables have been calculated for $\beta=0.84$ and $\alpha=0.2$ as a representative case. For the sake of brevity, the plots of the stream lines and pressures for the systems with a viscoelastic matrix are not shown here. The contour plots of the pressure are nearly indistinguishable from those for systems with a viscoelastic droplet. In addition, the plots of the stream lines show that the recirculation zone at the front and rear of the droplet is extended over the same portion of the gap for a Newtonian droplet in a viscoelastic matrix as compared to a viscoelastic droplet in a Newtonian matrix.

The contour plots of the viscoelastic stress and the dimensionless elongation rate for Newtonian droplets in a viscoelastic matrix are shown in Fig. 8 for different confinement ratios. From Figs. $8(\mathrm{a})-8(\mathrm{c})$ it is clear that, similar to the results for a system with a viscoelastic droplet

(Fig. 5), the maximum value of the viscoelastic stress increases when the confinement ratio is increased. In addition, it can be seen that the area around the droplet where substantial viscoelastic stresses are present is significantly extended if the droplet becomes more confined. However, the position of the maximum viscoelastic stress, which is slightly above the droplet tip, remains exactly the same, independent of the confinement ratio.

The contour plots of the dimensionless elongation rate are given in Figs. 8(d) - 8(f). These contour plots show that the maximum dimensionless elongation rate increases with confinement ratio, as was shown in Fig. 7. The position of the maximum with respect to the droplet remains however the same. The highest viscoelastic stress (Figs. 8(a)-8(c)) is present some distance downstream of the maximum elongation rate. This is in agreement with the results of the $2 \mathrm{D}$ simulations of Yue et al. [46] for bulk conditions and it reflects the finite relaxation time of the polymer molecules. An increase of the maximum value of the viscoelastic stress was also observed in bulk conditions, when increasing the values of De or Wi [17]. However, in that case the increase of the viscoelastic stress is coupled to a shift of the position of the maximum value higher upwards along the droplet contour. This is a reflection of the higher relaxation time of the polymer molecules due to the increase of Wi. The higher values of the viscoelastic stress combined with a shift of the position of the maximum, resulted in a reduction of the droplet deformation (Figure 14 in [17]). However, the increase of the maximum viscoelastic stress in 


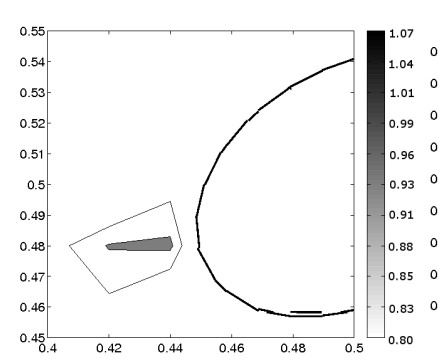

(a) $2 \mathrm{R} / \mathrm{H}=0.09$

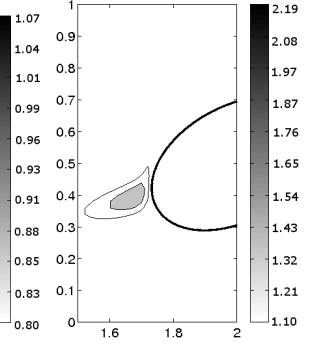

(b) $2 \mathrm{R} / \mathrm{H}=0.44$

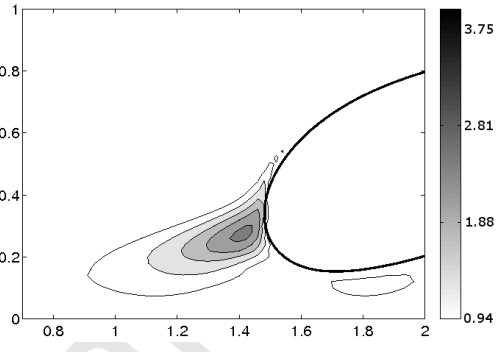

(c) $2 \mathrm{R} / \mathrm{H}=0.76$

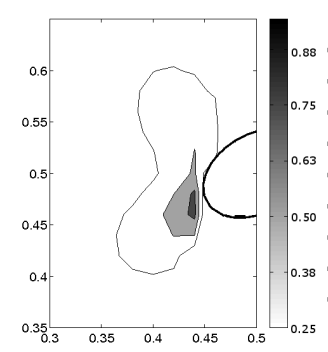

(d) $2 \mathrm{R} / \mathrm{H}=0.09$

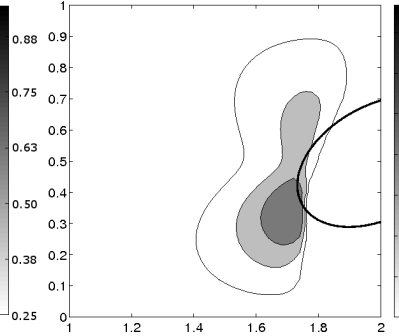

(e) $2 \mathrm{R} / \mathrm{H}=0.44$

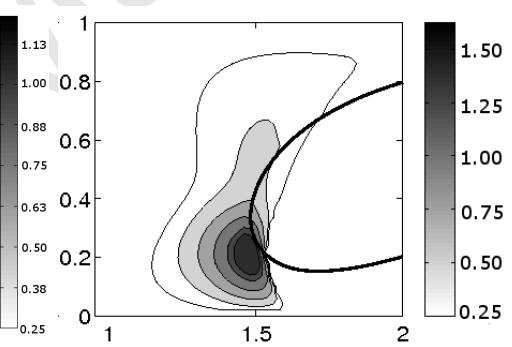

(f) $2 \mathrm{R} / \mathrm{H}=0.76$

FIG. 8: Newtonian droplet in a viscoelastic matrix at $\mathrm{Ca}=0.2$ and $\lambda=1.5$, Giesekus model with $\beta=0.84$ and $\alpha=$ 0.2; (a)-(b)-(c) Contour plots of trace (T) in the x-z cross-section, stress values are in Pa, (d)-(e)-(f) Contour plots of the dimensionless elongation rate $\left(\dot{\varepsilon} \cdot \tau_{P}\right)$. 
the matrix due to confinement causes an increase of the droplet deformation as compared to the Newtonian reference case. This conclusion is derived from a comparison between the results in Table 2 for systems with a viscoelastic matrix and in Fig. 2 for systems with a viscoelastic droplet. It can be seen there that the droplet deformation parameter in bulk conditions is 0.19 for a viscoelastic matrix (Giesekus model with $\beta=0.84$ and $\alpha=0.2$ ) compared to 0.23 for a viscoelastic droplet, whereas at $2 \mathrm{R} / \mathrm{H}=0.75$ this becomes 0.33 compared to 0.32 for respectively a viscoelastic matrix and a viscoelastic droplet.

The matrix fluid in the experimental system has a spectrum of relaxation times. Therefore, the viscoelastic stress is expected to be spread over a larger zone from the droplet tip upwards along the droplet contour. Based on the results in Figs. 8(a) - 8(c) an increase of the viscoelastic stress in this complete zone is expected, which might contribute to the fact that in the experimental data, matrix viscoelasticity reduces the droplet deformation for all confinement ratios. In addition, it should be kept in mind that the elongational viscosity was reduced to the same order of magnitude as the shear viscosity in the simulations, whereas the Trouton ratio in the experimental fluid is approximately $3.10^{4}$ [17].

Fig. 8 shows that the elongation rates inside the droplet are much lower than outside the droplet, with only a very small region of high elongation rates close to the droplet tip. For the systems with a viscoelastic droplet (Section 4.1), the contour plots of the elongation rate are similar to those for the systems with a viscoelastic matrix. However, at the same De, the value of Wi for the systems with a viscoelastic matrix with $\beta=0.84$ is substantially higher than that for a system with a viscoelastic droplet. Therefore, in the case of a viscoelastic droplet at $\mathrm{Ca}=0.2$, the elongation rate inside the droplet remains well below the critical value of 0.5 for divergence of the elongational viscosity at all confinement ratios.

For bulk shear flow, it has been shown that at high capillary numbers, viscoelasticity of the matrix phase can cause overshoots in the transient droplet deformation and droplet orientation after startup of shear flow [17, 18, 22]. Yue et al. [45] attributed this behavior to a mismatch of two time scales: the emulsion time $\tau_{E}$ for droplet deformation and the relaxation time $\tau_{P}$ of the polymers in the viscoelastic matrix phase. Since a reduction of the droplet deformation due to matrix viscoelasticity only occurs after a time interval of the order $\tau_{P}$, this causes an overshoot in the droplet deformation when $\tau_{E}$ is substantially shorter than $\tau_{P}$. Figure 6 shows that the kinetics of the droplet deformation after startup of shear flow speeds up due to confinement. This 


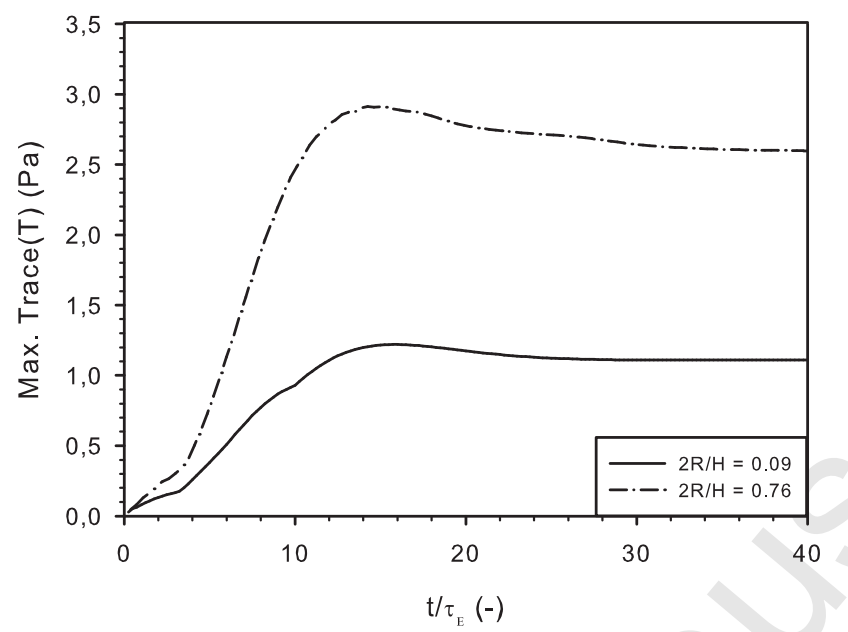

FIG. 9: Maximum value of the trace(T) as a function of time for systems with a Newtonian droplet in a Giesekus matrix fluid with $\beta=0.84$ and $\alpha=0.2$.

faster kinetics might change the ratio of the droplet to the polymer relaxation time and could thus induce overshoots in droplet deformation in confined shear flow. In order to investigate this in more detail, the evolution of the maximum value of the trace $(\mathbf{T})$ as a function of time has been studied. Results for Newtonian droplets in a viscoelastic matrix are given in Fig. 9 for two different confinement ratios. This figure shows that confinement results in a faster buildup of the viscoelastic stresses, which corresponds to the faster dynamics of the droplet deformation, that is presented in Fig. 6. From a comparison between Figs. 6 and 9 it can be concluded that the faster development of the droplet deformation in confinement as compared to bulk conditions is accompanied by a more or less equal increase in the growth speed of the viscoelastic stresses. This simultaneous buildup of the viscoelastic stresses and the droplet deformation results in a monotonous evolution towards a steady state droplet shape. However, confinement introduces a slight overshoot in the evolution of the maximum viscoelastic stress that is not caused by, nor results in an over- or undershoot in droplet deformation.

In more concentrated blends, a droplet is surrounded by other droplets and frequently interacts and collides with its neighboring droplets. When droplets in a viscoelastic matrix come close together, overlap of the regions with high viscoelastic stresses around each droplet will influence 
their interaction. Therefore, matrix viscoelasticity is expected to significantly enhance droplet interactions in confined blends. In addition, although less severe than solid walls, neighboring droplets in a blend also cause a sort of confinement, which might expand the zones with significant viscoelastic stresses, even in bulk conditions, and thus might alter the droplet interactions in concentrated blends. In blends with viscoelastic droplets on the other hand, the viscoelastic stresses are restricted within the viscoelastic fluid. Therefore, matrix viscoelasticity is expected to have a significant influence on droplet interactions and coalescence in concentrated blends whereas the effects of droplet viscoelasticity on these processes are expected to be rather limited.

\section{Concluding remarks}

An experimental and numerical study of droplet dynamics after startup of shear flow in systems with one viscoelastic component is conducted for bulk and confined conditions. In the experiments, the viscoelastic phase is a highly elastic Boger fluid, whereas fluids that obey a single-mode Oldroyd-B or Giesekus constitutive equation are used in the numerical modeling. Results are presented for blends with a viscosity ratio of 1.5 and flow capillary number 0.2 . The numerical results allow us to view the evolution of pressure and viscoelastic stresses, and the interesting correlation of the elongation rate with limiting behavior of the Oldroyd-B model.

Confinement is shown to accelerate the kinetics of droplet deformation independent of which phase is viscoelastic. Both for systems with a viscoelastic matrix and for systems with a viscoelastic droplet, the droplet deformation and orientation towards the flow direction increase when the confinement ratio is increased. However, for the studied experimental system, the droplet deformation in a viscoelastic Boger fluid matrix is smaller than that in a Newtonian matrix, for all studied confinement ratios. The simulations show that confinement increases the pressure just inside the droplet tip and the visualization of the streamlines shows that a stagnation point is generated just outside the droplet tips where a highly elongational flow occurs close to the interface. These observations are in agreement with the results for Newtonian droplet/matrix pairs $[12,15]$. In addition, we found that confinement substantially increases the elongation rates. In the case of a viscoelastic droplet, the purely rotational flow inside the droplet yields relatively low levels of viscoelastic stresses. When the matrix is viscoelastic, the strongly elongational flow which follows the dividing streamline just outside the droplet tip generates higher elastic stresses, and the region of maximum elastic stress expands with confinement. This phenomenon 
has implications for droplet interactions and droplet coalescence in concentrated blends in which droplets are confined between their neighbors. In such systems, the effect of matrix viscoelasticity is expected to dominate over that of droplet viscoelasticity. The occurrence of high gradients in viscoelastic stresses generated by the Oldroyd-B model for highly confined droplets is a barrier, given the numerical resolution, and therefore, one compromise is to reduce the elongational viscosity with the use of the Giesekus parameter. However, this impedes quantitative agreement with the experimental data for Boger fluids that possess a high elongational viscosity. It is shown that the sensitivity of the droplet deformation to the used constitutive equation is higher in confined conditions as compared to bulk conditions. Therefore, further refinement and validation of numerical codes for confined droplet dynamics in the presence of viscoelasticity is needed to enable modeling of shear-induced morphology development in confined blends.

\section{Acknowledgements}

R. Cardinaels is indebted to the Research Foundation - Flanders (FWO) for a Ph. D. Fellowship. This work was funded by NSF-DMS 0907788 and the TeraGrid Large Resource Allocations grant MCA08X019. We thank A. Avila (CEMCC, Universidad de La Frontera, Chile) and VT-ARC for computational resources. Part of the research was conducted at the Institute for Mathematics and Its Applications, the University of Minnesota, Minneapolis, MN.

\section{References}

[1] L. Shui, J. C. T. Eijkel, A. van den Berg, Multiphase flow in microfluidic systems - control and applications of droplets and interfaces, Adv. Colloid Int. Sci. 133 (2007) 35-49.

[2] H. A. Stone, S. Kim, Microfluidics: Basic issues, applications, and challenges, AIChE Journal 47 (2001) 12501254.

[3] H. A. Stone, A. Stroock, A. Ajdari, Engineering flows in small devices microfluidics toward a lab-on-a-chip, Ann. Rev. Fluid Mech. 36 (2004) 381-411.

[4] T. M. Squires, S. Quake, Microfluidics: Fluid physics at the nanoliter scale, Rev. Modern Physics 77 (2005) 977-1026.

[5] P. V. Puyvelde, A. Vananroye, R. Cardinaels, P. Moldenaers, Review on morphology development of immiscible blends in confined shear flow, Polymer 51 (2008) 5363-5372.

[6] P. E. Arratia, J. P. Gollub, D. Durian, Polymeric filament thinning and breakup in microchannels, Phys. Rev. E 77 (2008) 036309.

[7] D. J. E. Harvie, J. J. Cooper-White, M. Davidson, Deformation of a viscoelastic droplet passing through a microfluidic contraction, J. Non-Newt. Fluid Mech. 155 (2008) 67-79. 
[8] J. Husny, J. Cooper-White, The effect of elasticity on drop creation in t-shaped microchannels, J. Non-Newt. Fluid Mech. 137 (2006) 121-136.

[9] B. Steinhaus, A. Q. Shen, R. Sureshkumar, Dynamics of viscoelastic fluid filaments in microfluidic devices, Phys. Fluids 19 (2007) 073103.

[10] A. Vananroye, R. Cardinaels, P. V. Puyvelde, P. Moldenaers, Effect of confinement and viscosity ratio on the dynamics of single droplets during transient shear flow, J. Rheol. 52 (2008) 1459-1475.

[11] V. Sibillo, M. Simeone, S. Guido, F. Greco, P. Maffettone, Start-up and retraction dynamics of a Newtonian drop in a viscoelastic matrix under simple shear flow, J. Non-Newtonian Fluid Mech. 134 (2006) 27-32.

[12] P. Janssen, P. Anderson, Boundary-integral method for drop deformation between parallel plates, Phys. Fluids 19(4) (2007) 043602.

[13] P. Janssen, P. Anderson, A boundary-integral model for drop deformation between two parallel plates with non-unit viscosity ratio drops, J. Comp. Phys. 227 (2008) 8807-8819.

[14] Y. Renardy, The effect of confinement and inertia on the production of droplets, Rheol. Acta 46(4) (2007) 521-529.

[15] A. Vananroye, P. J. A. Janssen, P. D. Anderson, P. C. J. V. Puyvelde, P. Moldenaers, Microconfined equiviscous droplet deformation, comparison of experimental and numerical results, Phys. Fluids 20 (2008) 013101.

[16] R. Cardinaels, K. Verhulst, P. Moldenaers, Influence of confinement on the steady state behaviour of single droplets in shear flow for immiscible blends with one viscoelastic component, J. Rheol. 53 (2009) 1403 - 1424.

[17] K. Verhulst, R. Cardinaels, P. Moldenaers, Y. Renardy, S. Afkhami, Influence of viscoelasticity on drop deformation and orientation in shear flow. Part 1: Stationary states, J. Non-Newtonian Fluid Mech. 156 (2009) 29-43.

[18] K. Verhulst, P. Moldenaers, M. Minale, Drop shape dynamics of a Newtonian drop in a non-Newtonian matrix during transient and steady shear flow, J. Rheol. 51 (2007) 261-273.

[19] S. Guido, M. Simeone, F. Greco, Deformation of a Newtonian drop in a viscoelastic matrix under steady shear flow. Experimental validation of slow flow theory, J. Non-Newtonian Fluid Mech. 114 (2003) 65-82.

[20] V. Sibillo, S. Guido, F. Greco, P. Maffettone, Single drop dynamics under shearing flow in systems with a viscoelastic phase, in: Times of Polymers, Macromolecular Symposium 228, Wiley-VCH Berlin, 2005, pp. 31-39.

[21] W. Lerdwijitjarud, A. Sirivat, R. G. Larson, Influence of dispersed-phase elasticity on steady-state deformation and breakup of droplets in simple shearing flow of immiscible polymer blends, J. Rheol. 48(4) (2004) 843-862.

[22] P. L. Maffettone, F. Greco, M. Simeone, S. Guido, Analysis of start-up dynamics of a single drop through an ellipsoidal drop model for non-Newtonian fluids, J. Non-Newtonian Fluid Mech. 126 (2005) 145-151.

[23] K. Verhulst, R. Cardinaels, P. Moldenaers, S. Afkhami, Y. Renardy, Influence of viscoelasticity on drop deformation and orientation in shear flow. Part 2: Dynamics, J. Non-Newtonian Fluid Mech. 156 (2009) 44-57.

[24] R. Cardinaels, P. Moldenaers, Relaxation of fibrils in blends with one viscoelastic component: From bulk to confined conditions, J. Polym. Sci. Part B 48 (2010) 1372-1379.

[25] M. Minale, S. Caserta, S. Guido, Microconfined shear deformation of a droplet in an equiviscous non-Newtonian immiscible fluid: Experiments and modeling, Langmuir 26 (2010) 126-132.

[26] S. Afkhami, P. Yue, Y. Renardy, A comparison of viscoelastic stress wakes for $2 \mathrm{~d}$ and $3 \mathrm{~d}$ Newtonian drop deformation in a viscoelastic matrix under shear, Phys. Fluids 21 (2009) 072106.

[27] N. Aggarwal, K. Sarkar, Deformation and breakup of a viscoelastic drop in a Newtonian matrix under steady shear, J. Fluid Mech. 584 (2007) 1-21. 
[28] N. Aggarwal, K. Sarkar, Rheology of an emulsion of viscoelastic drops in steady shear, J. Non-Newt. Fluid Mech. 150 (2008) 19-31.

[29] D. Khismatullin, Y. Renardy, M. Renardy, Development and implementation of VOF-PROST for 3d viscoelastic liquid-liquid simulations, J. Non-Newtonian Fluid Mech. 140 (2006) 120-131.

[30] S. Mukherjee, K. Sarkar, Effects of viscosity ratio on deformation of a viscoelastic drop in a Newtonian matrix under steady shear, J. Non-Newtonian Fluid Mech. 160 (2009) 104-112.

[31] Y. Renardy, Drop oscillations under simple shear in a highly viscoelastic matrix, Rheol. Acta 47(1) (2008) 89-96.

[32] S. Guido, M. Simeone, M. Villone, Diffusion effects on the interfacial tension of immiscible polymer blends, Rheol. Acta 38 (1999) 287-296.

[33] F. Greco, Drop deformation for non-Newtonian fluids in slow flows, J. Non-Newtonian Fluid Mech. 107 (2002) $111-131$.

[34] J. Li, Y. Renardy, M. Renardy, Numerical simulation of breakup of a viscous drop in simple shear flow through a volume-of-fluid method, Phys. Fluids 12 (2000) 269-282.

[35] Y. Renardy, M. Renardy, T. Chinyoka, D. B. Khismatullin, A viscoelastic VOF-PROST code for the study of drop deformation, Proceedings of the ASME Heat Transfer/Fluids Engineering Summer Conference Paper No. HT-FED2004-56114 (2004).

[36] T. Chinyoka, Y. Renardy, M. Renardy, D. B. Khismatullin, Two-dimensional study of drop deformation under simple shear for Oldroyd-B liquids, J. Non-Newtonian Fluid Mech. 130 (2005) 45-56.

[37] Y. Renardy, M. Renardy, PROST: a parabolic reconstruction of surface tension for the volume-of-fluid method, J. Comput. Phys. 183(2) (2002) 400-421.

[38] N. Aggarwal, K. Sarkar, Effects of matrix viscoelasticity on viscous and viscoelastic drop deformation in a shear flow, J. Fluid Mech. 601 (2008) 63-84.

[39] S. Afkhami, M. Bussmann, Height functions for applying contact angles to 3D VOF simulations, Int. J. Numer. Meth. Fluids 61 (2009) 827-847.

[40] S. Afkhami, M. Bussmann, Height functions for applying contact angles to 2D VOF simulations, Int. J. Numer. Meth. Fluids 57 (2008) 453-472.

[41] A. Vananroye, P. J. A. Janssen, P. D. Anderson, P. C. J. V. Puyvelde, P. Moldenaers, Microconfined equiviscous droplet deformation, comparison of experimental and numerical results, Phys. Fluids 20 (2008) 013101.

[42] K. Verhulst, R. Cardinaels, P. Moldenaers, Y. Renardy, S. Afkhami, Influence of viscoelasticity on drop deformation and orientation in shear flow. Part 1: Stationary states, J. Non-Newtonian Fluid Mech. 156 (2009) 29-43.

[43] C. Chen, R. Cardinaels, P. Moldenaers, Effect of confinement on droplet coalescence in shear flow, Langmuir 25 (22) (2009) 12885-12893.

[44] V. Sibillo, G. Pasquariello, M. Simeone, V. Cristini, S. Guido, Drop deformation in microconfined shear flow, Phys. Rev. Lett. 97 (2006) 054502.

[45] P. Yue, J. J. Feng, C. Liu, J. Shen, Transient drop deformation upon startup of shear in viscoelastic fluids, Phys. Fluids 17 (2005) 12310/1-7.

[46] P. Yue, J. J. Feng, C. Liu, J. Shen, Viscoelastic effects on drop deformation in steady shear, J. Fluid Mech. 540 (2005) 427-437.

[47] V. Tirtaatmadja, T. Sridhar, Comparison of constitutive equations for polymer solutions in uniaxial extension, J. 
Rheol. 39 (1995) 1133-1160.

[48] B. Thomases, M. Shelley, Emergence of singular structures in oldroyd-b fluids, Phys. Fluids 19 (2007) 103103-1 $-103103-12$.

[49] Y. Renardy, M. Renardy, Instability due to second normal stress jump in two-layer shear flow of the Giesekus fluid, J. Non-Newtonian Fluid Mech. 81 (1999) 215-234.

[50] J. J. Magda, J. Lou, S. G. Baek, K. Devries, Second normal stress difference of a boger fluid, Polymer 32 (1991) 2000-2009.

[51] L. Levitt, C. W. Macosko, S. D. Pearson, Influence of normal stress difference on polymer drop deformation, Polym. Eng. Sci. 36 (1996) 1647-1655.

[52] M. Alcoutlabi, S. G. Baek, J. J. Magda, X. Shi, S. A. Hutcheson, G. McKenna, A comparison of three different methods for measuring both normal stress differences of viscoelastic liquids in torsional rheometers, Rheol. Acta 48 (2009) 191-200.

[53] S.-G. Baek, J. J. Magda, Monolithic rheometer plate fabricated using silicon micromachining technology and containing miniature pressure sensors for N1 and N2 measurement, J. Rheol. 47 (2003) 1249-1260.

[54] H. H. Hu, O. Riccius, K. P. Chen, M. Arney, D. Joseph, Climbing constant, second-order correction of Trouton's viscosity, wave speed and delayed die swell for M1, J. Non-Newt. Fluid Mech. 35 (1990) 287-307.

[55] M. Keentok, A. G. Georgescu, A. A. Sherwood, R. Tanner, The measurement of the second normal stress difference for some polymer solutions, J. Non-Newt. Fluid Mech. 6 (1980) 303-324. 